\title{
WiFi Management for Dense Area
}

\author{
Xinyan Guo 1, a * \\ ${ }^{1}$ Business School of Sichuan University, Chengdu China \\ asindystory@163.com
}

Keywords: WiFi, Management, Dense area, High throughput

Abstract. WiFi is every where now. In many places such as: home, office and public areas, the user could easily access the network, surf the internet, view the online video clips, and download big data through WiFi. However, in current WiFi infrastructure, the devices within the vicinity have to share the channel in a time sharing fashion. Thus the network throughput decreases linearly with the number of active devices. With more and more applications going through wireless, how to get the higher throughput in these dense area, become a hot research topic in the WiFi area.

To solve this throughput challenge in dense area, research institute and IT worked together and proposed one WiFi management protocol. The main idea of this new protocol is to increase the throughput via higher spatial reuse. In the new protocol, the devices will reserve the normal space area through the handshake package (RTS/CTS), same as the legacy protocol. But after the initial device reserves the channel, it will reduce its data transmission power to decrease the wireless signal interference, and meanwhile invite the nearby devices to join the transmission. So the participant devices would reuse the channel/space which the initial device reserves, and more devices could transmit the data at the same time.

We implemented and enable this WiFi management protocol on wireless card. And in the field trial with 6 laptops ( 3 pairs transmitting data at the same time), the new protocol got above $80 \%$ throughout increase comparing to the legacy WiFi protocol. And the benefit will be increased linearly with the number of the participant devices in the environment. This new WiFi management protocol will help increase the overall throughput in dense area dramatically, and it will also enable our IT to deploy the throughput-demanding applications, like wireless docking, in the company.

\section{Introduction}

The wireless dense area is always the bottleneck for WiFi transmission and result in low transmission efficiency. It provides the bad user experience.

Previously, most common solutions can be summarized in 2 ways. One is to reduce the transmission power to decrease the radio signal interference. The other one is to improve the sensitivity level to enforce WiFi stations become more activity in strong wireless interference. But the drawbacks are also obvious. For the first one, the protected area of transmission stations will become weak so that can't resist interference from other full power stations working legacy protocol. For the second one, each station will become more active to transmit data that could bring more radio signal interference to the environment to influence other stations, after that the total throughput can't improve actually.

This paper studies a particular low power protocol with special handshake mechanism to address the throughput bottleneck in dense area without above shortcomings. And what's more, the new protocol also support to remove excessive data transmission power even to $30 \mathrm{~dB}$ (1/1000 data packet power in RF signal) due to the low power data transmission and power adjustment/control mechanism. So this is another outstanding feature to extend the battery life. This feature is especially meaningful in mobile device.

The more significant value in this protocol is only need to modify in the source code WiFi driver, but no need to modify in physical design in WiFi card. So we can call it a low cost solution, but can provide us more benefit. This new protocol can be applied to WiFi environment, and also can propose in WiFi standards. 
The rest of this paper is described as follows. The methodology includes the fundamental principle of this new protocol in section 2. Section 3 describes the implementation details and demonstrate prototype on WiFi card. Section 4 will describe the challenge in engineering to implement this proposal. Section 5 provides the evaluation result in actual dense area and compares the throughput improvement between new and legacy protocol. Section 6 will give a summary conclusion. Figure 1 shows the difference between traditional and new WiFi management protocol.

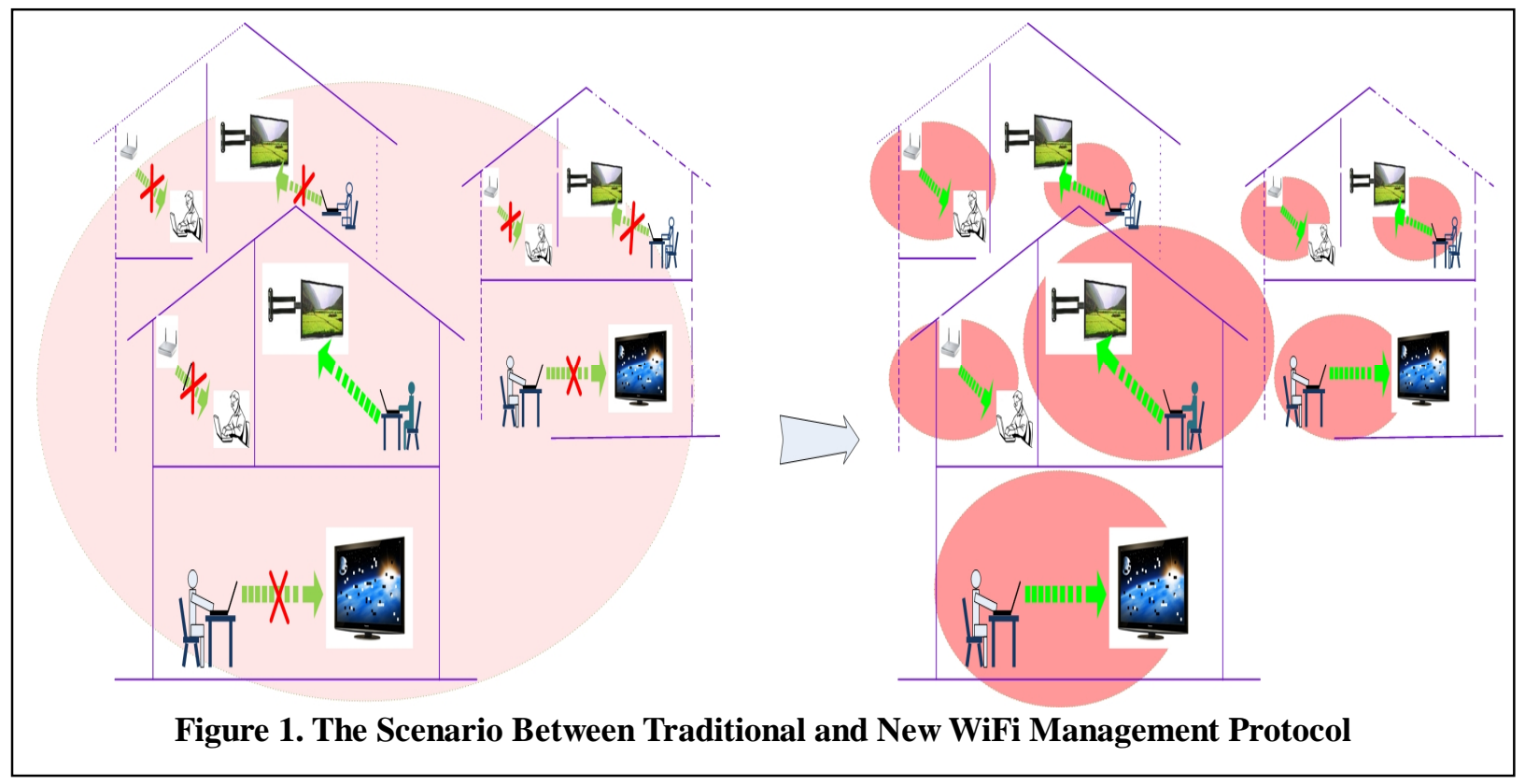

\section{Methodology}

As the WiFi legacy transmission protocol, only one pair of stations can occupy the channel through contention window (Backoff first, then occupy the channel by RTS-CTS control packets). Just this pair can use the occupied space to transmit data packet in its transmission period. We call it initial pair. All other stations will hold itself during this time not to produce wireless interference, avoid to interfere the initial pair transmission. So the design prototype in legacy protocol only allows one pair to use the same space for data transmission at one time. The space usage ratio is too low. The total throughput for all stations in the same space area is limited, and the average bandwidth on each station could decrease lower with the increased stations in the same area.

This paper is to devise a new protocol to address the issue of throughput bottleneck in dense area. The key point is to improve the space use ratio to increase the total throughput and the principle is to reduce the power in data packet to decrease the wireless signal interference first. Then two new control packets created with a new handshake communication mechanism for the participation of other stations.

In general, all the control packets are in full power to occupy space area to protect each single station, but all data packets are in low power to decrease the signal interference to enforce more stations can join in the transmission at the same time. So all of the stations are compatible with legacy stations but can gain much throughput in new protocol.

This new protocol is especially suit for the layout of WiFi in apartment building and wireless building.

\section{Implementation}

The new WiFi management implementation is described as follows. Please refer the details of each stage. 


\section{Space Contention}

As the current protocol, stations should also use full power RTS, CTS control packets before data transmission for two purposes. One is to handshake between the source station and destination station to establish the communication in initial pair.

\section{Construct New Control packets}

All other pairs will be reserved by the initial pair through contention window. But in order to improve the total throughput, we should activate the reserved stations to join in and transmit at the same time. So we devised two new control packets CFLP (Call For Low Power) and CFLP_CTS (reuse Self_CTS) in the new protocol to ask all reserved stations to join in by CFLP, and all these reserved stations could response by CFLP_CTS to prepare the participant transmission.

After the above handshake, all the reserved stations have been activated. That means CFLP as a broadcast packet to notify all stations clear its own reserved timer to zero and ready to start the transmission Each station will response a full power CFLP_CTS once receive the CFLP packet from initial pair. This response has two purposes, one is to tell initial pair there are some other stations could participate in this transmission period which belongs to the initial pair. The second is to protect itself as Self_CTS due to lack of RTS/CTS to protect itself at the beginning, then the participant pair could transmit data in a safe area.

\section{Data Transmission}

The initial pair will start data transmission in low power after CFLP -> Self_CTS handshake. The low power data packet could produce low interference of wireless signal, so the initial pair only occupies a small area to transmit data packet. The other space could be utilized by other participant pairs for data transmission. All other participant pairs could also use low power to transmit data. So each pair just occupies a small space (compare with legacy protocol in full power transmission with a large space area). All stations could reuse the same space to transmit data at the same time to increase the total throughput. In the definition in legacy protocol, the full power data transmission needs to cover at least 150 feet for the safe wireless transmission. But in the new protocol, each station can reduce 20 - 30DB (1/100 to $1 / 1000$ ratio compared with legacy) data transmission power, it could decrease the wireless signal interference and the covered space can be reduced to 15 feet (1/10 radius compared with legacy protocol). So all the transmission pairs outside 15 feet can join in and transmit data at the same time without interference to each other. This technology is very suitable for the wireless transmission layout in offices building.

The following diagram describes data transmission in new protocol. The data interference in each pair can be limited in a small area about 15 feet (in red circle) but the protected area is also 150 feet (in gray circle) as the same as before to resist the interference from other full power stations working in legacy protocol.

By the way, the data transmission power can be adjusted in new protocol. It depends on the transmission distance. So the red circle can be scaled.

If the distance is short in every two cubicles, we could reduce more power to include more participant pairs. If the distance between source and destination station become longer, we could improve data power to keep high MCS rate (high speed).

\section{Timing Sequence}

The transmission timing sequence should be modified to suit the new protocol.

Step 1: Only one station can occupy the space during the contention window, other stations will be reserved by this station.. (Initial pair, RTS-CTS)

Step 2: Initial pair broadcast CFLP packet to the space to invite other reserved stations to join in during this transmission period. All other stations will response the CLFP_CTS once receiving the CFLP and prepare to transmit data to reuse the space. 
Step 3: All data transmission use low power to reduce the interference and reuse the space to improve the total throughput.

Step 4: The control packet of CF-End (Contention Frame End) will be removed from initial pair in new protocol in order to let all participant pairs to complete transmission even if the initial pair has completed earlier.

\section{Implementation in Engineering}

This new protocol is not only verified in simulation via modelling, and also test in engineering. Firmware modification - This is the lowest level of the driver and in charge of PHY layer in WiFi. The actual transmission of all packets (include control/management/data packets) is in this layer and modulate in physical format to transmit. We created two new control packets in this layer and add them in the timing sequence of data transmission. We also change the states machine in firmware to meet the new protocol and adapt different roles in one station.

NDIS miniport modification - In Windows Operating System, the miniport layer in NDIS is treated as the driver of WiFi physical device. We modify the sensitivity mechanism to improve the participant ratio and implement some new commands to control WiFi behavior for applications.

NDIS protocol - In Windows Operating System, the protocol layer in NDIS is treated as the driver of WiFi logic device. The application can only access logic device to control WiFi hardware (physical device). We implement a new protocol driver here to access the new features conveniently in NDIS miniport for applications.

\section{Challenges}

In the development in engineering, there are some issues should be fixed.

\section{Participant Ratio}

There are several MCS rate to transmit wireless packet in the mode of OFDM modulation defined in IEEE 802.11n. Due to the background noise or other interference in actual test environment, a few stations in this area can't receive or demodulate correctly of the CFLP packet from initial station sometimes. So it will lead to less participant ratio not as the expectation. A valid way to send CFLP more distinct is to decrease its MCS rate by low modulation mode.

The MCS rate of control packets defined $<=24 \mathrm{Mbps}$. And we observe the actual MCS rate in engineering card almost $12 \mathrm{Mbps}$ or $24 \mathrm{Mbps}$ (depends on the environment). If we want to increase the participant ratio (extend reserved area, or demodulate CFLP correctly by reserved stations in noise environment), we could reduce the CFLP rate to the lowest MCS (6Mbps) rate. After the experiment, we can observe the participant ratio and total throughput have been improved.

\section{Data Power Adjustment}

The data transmission power depends the distance between source and destination stations.

If the data transmission power is fixed, it will consume more power while in short distance or without enough power to guarantee transmission correctly in long distance.

If the data transmission power sets too high, the reserved space in each pair will become large to influence other stations to participate in.

If the data transmission power sets too low, the MCS rate will be reduced due to the SNR in destination side. So the throughput in each pair will reduce more to decrease the total throughput, even if more stations could participate in.

Table 1 at right side clearly describes the necessary power energy in RX side.

So we devise a power control mechanism to manage it dynamically in Figure 2 as below: 
Table 1: Power Energy With MCS Rate

\begin{tabular}{|c|c|c|c|c|c|}
\hline \multirow{2}{*}{$\begin{array}{l}\text { Min } \\
\text { RSSI } \\
\text { [dBm] }\end{array}$} & \multirow[t]{2}{*}{ MCS } & \multicolumn{2}{|c|}{$20 \mathrm{MHZ}$} & \multicolumn{2}{|c|}{$40 \mathrm{MHZ}$} \\
\hline & & NGI & SGI & NGI & SGI \\
\hline-87 & 0 & 6 & 7 & 13 & 15 \\
\hline-85 & 1 & 13 & 14 & 27 & 30 \\
\hline-84 & 2 & 19 & 21 & 40 & 45 \\
\hline-80 & 3 & 26 & 29 & 54 & 60 \\
\hline-72 & 4 & 39 & 43 & 81 & 90 \\
\hline-68 & 5 & 52 & 58 & 108 & 120 \\
\hline-64 & 6 & 58 & 65 & 121 & 135 \\
\hline-60 & 7 & 65 & 72 & 135 & 150 \\
\hline-87 & 8 & 13 & 14 & 27 & 30 \\
\hline-85 & 9 & 26 & 29 & 54 & 60 \\
\hline-84 & 10 & 39 & 43 & 81 & 90 \\
\hline-80 & 11 & 52 & 58 & 108 & 120 \\
\hline-72 & 12 & 78 & 87 & 162 & 180 \\
\hline-68 & 13 & 104 & 116 & 216 & 240 \\
\hline-64 & 14 & 117 & 130 & 243 & 270 \\
\hline-60 & 15 & 130 & 144 & 270 & 300 \\
\hline-87 & 16 & 20 & 22 & 41 & 45 \\
\hline-85 & 17 & 39 & 43 & 81 & 90 \\
\hline-84 & 18 & 59 & 65 & 122 & 135 \\
\hline-80 & 19 & 78 & 87 & 162 & 180 \\
\hline-72 & 20 & 117 & 130 & 243 & 270 \\
\hline-68 & 21 & 156 & 173 & 324 & 360 \\
\hline-64 & 22 & 176 & 195 & 365 & 405 \\
\hline-60 & 23 & 195 & 217 & 405 & 450 \\
\hline
\end{tabular}

\section{Result}

This technology is not only verified on simulation by Matlab, but also proved in WiFi card. Since this new protocol is focus on MAC and PHY layers of IEEE802.11 standard. So, the modification includes driver and firmware code of WiFi card. Since of the limited space in office and lab, there are 3 pairs of laptops layout to test the WiFi throughput in actual environment. The distance between every 2 pair is about 50 feet. And as the definition of IEEE802.11, the occupied space area for one station needs about 150 to 300 feet. So, it is much close than full power covered area compared with legacy protocol. Only refer to the test result on 3 pairs, the total throughput in legacy protocol is about $100 \mathrm{Mpbs}$, but above $200 \mathrm{Mbps}$ in new protocol. It is almost $100 \%$ throughput improvement than the legacy only in the test result of 3 pairs. If there is enough space to layout more pairs, so more stations could participate in at the same time to result in the total throughput with much more improvement. We build the below test environment, and the test result is published in figure 3:

- RF is in MIMO2

- Channel bandwidth is $20 \mathrm{MHz}$

- Data MCS rate by the highest modulation is $130 \mathrm{Mbps}$

- Throughput limitation in one channel is $108 \mathrm{Mbps}$. 


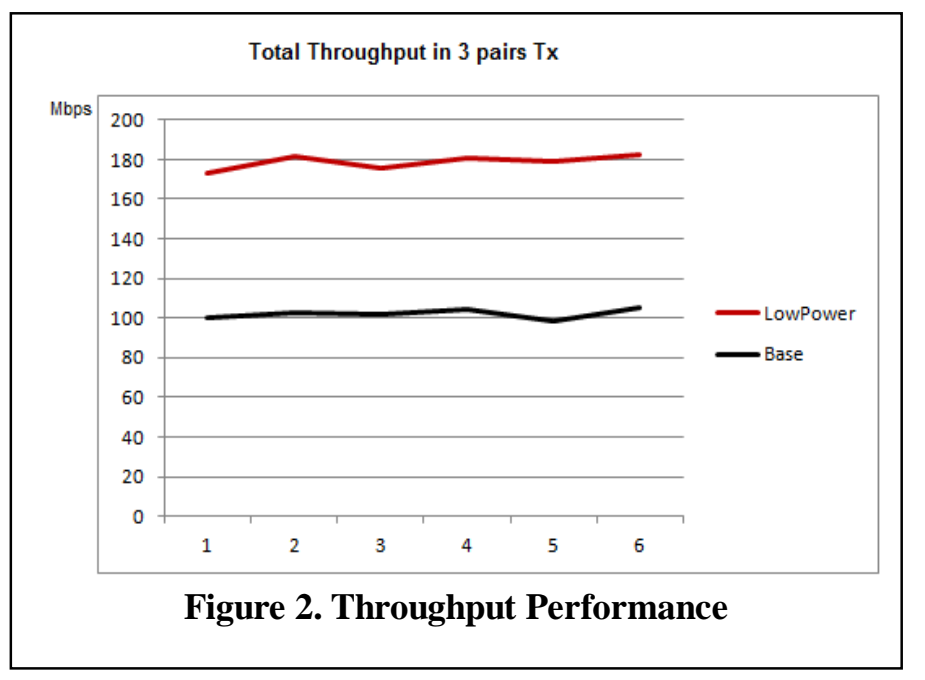

\section{Conclusion}

As shown in the above result, this new WiFi management will get almost $80 \%$ throughput improvement while 3 pairs are transmitting the data at the same time, comparing to the legacy protocol. And according to the mechanism of the protocol, the more devices join as the participant devices, the more benefits we will get by this new protocol. The benefit will be increased linearly with the number of the participant devices in the environment. So this new WiFi management will increase the overall throughput in dense area dramatically.

And this WiFi management is compatible with the existing / legacy protocol. The devices running the new protocol could co-exist with legacy devices, without the impact from the legacy devices or impact to these devices. Further, this is a low cost solution. All the enhancement of this protocol is on MAC layer; it could be implemented and enabled on existing HW without the need for HW change. So it will be easier to be adopted and deployed in current wireless environment or mobile devices. And it will be one good solution for our IT to deploy the throughput-demanding applications, like wireless docking, in the company.

Next step, research institute and IT are trying to propose this new protocol into Wireless card and are co-working with MCG standard team to integrate this new protocol as part of WiFi proposal for WiFi alliance.

\section{References}

[1] IEEE 802.11Standard for Wireless LAN Medium Access Control (MAC) and Physical Layer (PHY) specifications on http://standards.ieee.org/findstds/standard/802.11-1997.html

[2] IEEE 802.11-n Standard on http://standards.ieee.org/findstds/standard/802.11n-2009.html

[3] Malone, D. ; Clifford, P. ; Leith, D.J. MAC Layer Channel Quality Measurement in 802.11 on http://ieeexplore.ieee.org/xpl/articleDetails.jsp?tp=\&arnumber $=4115143 \&$ queryText $\% 3 \mathrm{D} \% 28802.11$ $\% 29$

[4] Jinkyu Lee ; Ikjun Yeom. Avoiding collision with hidden nodes in IEEE 802.11 wireless networks on

http://ieeexplore.ieee.org/xpl/articleDetails.jsp?tp=\&arnumber $=5288449 \& q u e r y T e x t \% 3 D \% 28802.11$ $\% 29$

[5] Microsoft Network Monitor and Analysis on http://www.microsoft.com/en-us/download/details.aspx?id=4865 